\title{
Coulisses
}

Revue de théâtre

1 | Printemps 1990

Varia

\section{Regards sur Hamlet}

Jean Jourd'heuil

\section{OpenEdition}

Journals

Édition électronique

URL : http://journals.openedition.org/coulisses/1548

DOI : 10.4000/coulisses.1548

ISSN : 2546-9460

\section{Éditeur}

Presses universitaires de Franche-Comté

\section{Édition imprimée}

Date de publication : 1 avril 1990

Pagination : 31-33

ISSN : 1150-594X

\section{Référence électronique}

Jean Jourd'heuil, « Regards sur Hamlet », Coulisses [En ligne], 1 | Printemps 1990, mis en ligne le 04 juillet 2017, consulté le 22 octobre 2019. URL : http://journals.openedition.org/coulisses/1548 ; DOI 10.4000/coulisses.1548

Ce document a été généré automatiquement le 22 octobre 2019

Coulisses 


\title{
Regards sur Hamlet
}

\author{
Jean Jourd'heuil
}

\section{NOTE DE L'ÉDITEUR}

Jean Jourdheuil, metteur en scène et dramaturge, a donné le 8 décembre 1989 une conférence sur le théâtre allemand contemporain. On rappellera que Jourdheuil a travaillé à la Schaubühne de Berlin avec Peter Stein, a traduit Brecht, Büchner, Kleist et Müller qu'il a contribué à faire connaître en France. Coulisses présente des extraits de cette conférence, notamment sur le thème de Hamlet où sont mises en parallèle les interprétations de P. Chéreau et H. Müller.

\section{Sur la tradition théâtrale à l'Est et à l'Ouest}

1 Peter Stein représente la tradition de l'Ouest où un metteur en scène travaille l'acteur au corps, lui demande d'être totalement capable d'incarner le personnage dans une optique stanislavskienne. Il faut se rappeler qu'en RFA le théâtre de Brecht - frappé d'une espèce d'ostracisme - était peu joué alors que paradoxalement le théâtre allemand que l'on connaît en France dans les années soixante est le théâtre de Brecht, principalement le Brecht épique de Mère Courage, Puntila. En Allemagne Fédérale, on était donc peu familiarisé avec le style de jeu narratif, avec des notions telle que la distanciation qui précisément marque la rupture avec l'inspiration stanislavskienne.

En RDA, le théâtre se situe plutôt dans une tradition brechtienne. On considère, selon la formule de Walter Benjamin, la scène comme un podium permettant de s'adresser directement au public. Contrairement aux acteurs de l'Ouest qui jouent sur euxmêmes, entre eux et regardent rarement la salle, les acteurs de l'Est ont une scène qui les met dans une situation où ils peuvent s'adresser aux spectateurs avec quelquefois une certaine brutalité. Cette relation avec le public provient du fait que le théâtre en RDA a une fonction totalement différente de celle qui prévaut en RFA. Le théâtre à l'Est remplit une fonction sociale, assure le rôle d'espace public que la presse, 
conventionnelle, ne joue plus alors qu'à l'Ouest, le théâtre, cérémonie bourgeoise, est davantage une célébration culturelle.

\section{Histoire de spectre}

Quand P. Chéreau monte Hamlet en France, on se demande quelle actualité cela peut avoir. Si on en juge par la presse évoquant le spectacle donné en Avignon, on est amené à penser que l'actualité, c'est le cheval, le cheval noir dont il a été beaucoup question. La chose est même allée très loin puisqu'on a eu le sentiment que seul le cheval avait du génie. Ce fait est révélateur : le spectre est dans Hamlet un problème clef, auquel est lié le cheval.

4 À l'époque où Shakespeare écrit, le spectre est au fond un problème de catholiques. Les protestants ne croient pas aux spectres. Lorsqu'on dit à Hamlet qu'il y a un spectre - il revient alors de Wittenberg, la ville de Luther -, celui-ci dit que cela mérite un peu de prudence car, qui sait, peut-être ce spectre est-il démoniaque, peut-être est- ce une apparition du diable ! Ce rapport au spectre permet le fonctionnement de la pièce. Or, qui croit encore aux spectres aujourd'hui? On est en présence d'une figure théâtrale totalement dévaluée. Chéreau a donc été obligé de doper cette figure et le moyen qu'il utilise est le cheval; il place le père sur un cheval et cela donne au spectre quelque chose d'imprévisible. Effectivement, le cheval tremble, bouge, tape du pied devant le spectre réintroduisant dans la pièce une sorte d'irrationalité.

5 Pour H. Müller, le spectre est ce qui a été tué. En Allemagne, qu'est-ce qui a été tué ? Les victimes ne manquent pas, celles du nazisme, du stalinisme. On a alors de quoi aborder la figure du spectre avec un autre background historique. De plus, il faut se souvenir de la tradition allemande de Hamlet. On peut dire que, grâce à Hamlet, Shakespeare est devenu le troisième classique allemand. Goethe, Schiller, Shakespeare constituent le trio classique, ce qui explique sans doute que Büchner et Kleist ne soient pas autre chose dans la culture allemande que des auteurs marginaux, Shakespeare occupant la place.

\section{Hamlet et Freud}

Chéreau tient sur Hamlet un discours extrêmement personnel soit existentiel soit psychanalytique. On est dans une situation où le seul discours critique possible actuellement en France est le discours psychanalytique. On a donc une interprétation très personnelle qui fonctionne à l'identification, Chéreau disant: "la solitude d'Hamlet, je la comprends bien et j'ai de bonnes raisons pour bien la comprendre ». Chéreau a effectivement de bonnes raisons passibles d'un commentaire analytique : son père meurt alors qu'il monte Hamlet tout comme le père de Shakespeare était mort peu avant l'écriture de la pièce. Hamlet est de ce point de vue une pièce placée sous le signe du deuil.

Dans un texte de 1977 intitulé Album de famille, H. Müller congédie d'emblée l'interprétation freudienne ainsi que toute approche structuraliste. Hamlet commence par coucher avec sa mère, ce qui casse la perspective freudienne. La pièce de Shakespeare est en effet construite sur le fait que Hamlet ne peut pas tuer Claudius puisque Claudius fait ce que lui, Hamlet, voudrait bien faire mais ne peut pas faire: 
c'est en quoi il est œdipien. L'important dans un traitement de Hamlet comme celui de Müller est que le dramaturge détruit sans vergogne le mythe de Hamlet en tant que mythe allemand en même temps qu'il fait jouer la pièce sur un rythme soutenu, à toute vitesse. Müller rétablit ainsi ce qui manque la plupart du temps aux mises en scène françaises où on n'arrive pas à prendre cela comme un jeu. Shakespeare prenait sa pièce comme un jeu et autre chose passait que le jeu alors qu'en France, on monte le tout d'une manière uniformément solennelle. La dimension du jeu disparaît et avec elle le tragique qui est l'essence même de la pièce.

\section{La tragédie de la vengeance}

Quand Shakespeare traite sa pièce, on est en 1600-1603, époque de la fin du règne d'Élizabeth où se pose avec acuité la question de la succession. Shakespeare est partisan de Jacques Ir d'Ecosse, fils de Marie Stuart exécutée sur ordre d'Élizabeth. Mais Jacques $I^{\text {er }}$ est aussi fils d'une femme qui s'est remariée avec l'assassin de son mari, et ce deux mois à peine après le drame. Jacques I ${ }^{\mathrm{er}}$ est l'héritier présomptif du trône. Si l'on songe au fait que parler de l'éventuelle complicité de la reine dans le meurtre de son mari, c'est commettre un crime de lèse-majesté à brève échéance et que d'autre part ne pas y faire allusion alors que tout le monde dans l'Angleterre puritaine considère que Marie Stuart est complice, c'est participer à cette complicité, on a le problème tel qu'il se pose à Shakespeare et à Hamlet. Le spectre intervient à plusieurs reprises sur ce problème, à la fois pour relancer la vengeance et toujours pour détourner la vengeance de la reine, disant en quelque sorte : " venge-moi mais ne t'en prends pas à ta mère ». Il $\mathrm{y}$ a dans la pièce une espèce de tabou de la reine. À partir de la tragédie de la vengeance, Shakespeare fait intervenir un formidable problème politique de l'époque. L'indécision d'Hamlet est la nécessaire indécision de Jacques Ier, roi en situation de clivage, paralysé, avec quelque chose d'un peu schizophrène ${ }^{1}$.

\section{BIBLIOGRAPHIE}

\section{Guvres de Heiner Müller publiées en France}

Hamlet-Machine, 1982, Minuit.

La Mission, 1982, Minuit.

La Comédie des femmes, 1984, Edilig.

Philoctète, 1985, Avant-Scène.

La Bataille, 1988, Minuit. 


\section{NOTES}

1. Jourdheuil précise qu'il s'agit là d'une analyse inspirée d'H. Müller qui la doit lui-même du juriste Carl Schmitt. 